\title{
SASKATCHEWAN CHRISTMAS MAMMAL COUNTS - 1995
}

Compiled by Wayne C. Harris, Saskatchewan Environment and Resource Management, 350 Cheadle Street West, Swift Current, SK. S9H 4G3

The number of mammal counts conducted this year dropped from the record 84 last year to 78 in 1995. The number of species recorded was 38 , down from last year's 42 .

More typical winter weather returned to the province in 1995. Unlike last year's count when a lack of snow made tracking conditions poor in much of the southwestern area, snow cover was generally good throughout the province. No new species were reported in 1995. Rare species included Swift Foxes reported from two counts, both within the areas designated for reestablishment of Swift Fox populations through re-introduction.

The most commonly reported species was White-tailed Deer, followed by Coyote and Red Fox. Most populations appeared stable. One exception was Red Fox that increased from $45 \%$ of the counts in 1994 to $64 \%$ in 1995 . White-tailed Deer were also up from $75 \%$ of counts to $84 \%$.

For weather, coverage and participants please refer to the Christmas Bird Count found elsewhere in this issue. Numbers appearing before the count location name in the tables refer to the location of the count on the map included with the bird count. In the tables, a numeral alone shows that the mammals were seen, while a letter preceding the number means that the number was inferred by the means defined below. A letter alone means that the species was present but estimating the numbers was impossible or that no attempt was made to do so.

$T=$ tracks

$L=$ active lodge or hut

$\mathrm{D}=$ dead animal found

$d=$ fresh diggings found

$S=$ smell or odour

$\mathrm{H}=$ heard

$+=$ present during the count period (16 December to 2 January) but not found on count day.

I wish to comment on errors that have appeared in the count compilation over the past two years. In 1993, 17 counts were added to the compilation after it left the editorial hands. Here all 17 counts were areas that had not conducted mammal counts and they were inserted with zero species seen inferring that counts were conducted when they were not. Last year (1994), for the first time ever, we had two counts conducted but zero species recorded; these were deleted, again after proofreading was complete. Furthermore, the numbers preceding the counts that provided location reference to the map were also changed so they referenced the wrong location. I wish to apologize to the compilers of these counts for these errors. 
Table 1-1: SASKATCHEWAN CHRISTMAS MAMMAL COUNTS

\section{SPECIES}

MASKED SHREW

SHREW species

EASTERN COTTONTAIL

NUTTALL'S COTTONTAIL

SNOWSHOE HARE

WHITE-TAILED JACK RABBIT

RICHARDSON'S GROUND SQUIRREL

BLACK-TAILED PRAIRIE DOG

GREY SQUIRREL

FOX SQUIRREL

AMERICAN RED SQUIRREL

NORTHERN FLYING SOUIRREL

AMERICAN BEAVER

DEER MOOUSE

GAPPER'S RED-BACKED VOLE

MUSKRAT

MEADOW VOLE

VOLE species

MOUSE species

NORWAY RAT

HOUSE MOUSE

AMERICAN PORCUPINE

COYOTE

WOLF

RED FOX

SWIFT FOX

RACCOON

FISHER

ERMINE

LONG-TAILED WEASEL

LEAST WEASEL

WEASEL species

AMERICAN MINK

AMERICAN BADGER

STRIPED SKUNK

RIVER OTTER

BOBCAT

MULE DEER

WHITE-TAILED DEER

DEER species

MOOSE

AMERICAN ELK

PRONGHORN

TOTAL SPECIES

\begin{tabular}{|c|c|c|c|c|c|c|c|c|c|}
\hline \multicolumn{10}{|c|}{ LOCATION AND DATE } \\
\hline 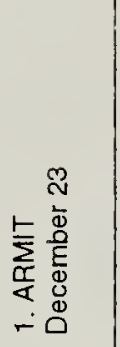 & 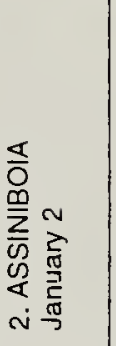 & 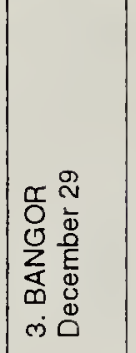 & 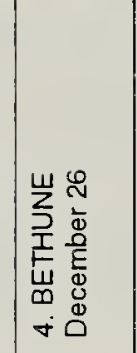 & 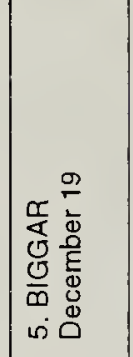 & 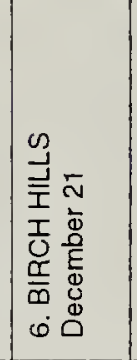 & 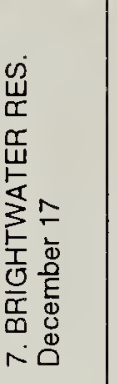 & 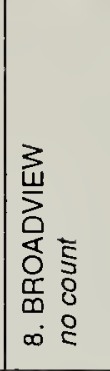 & 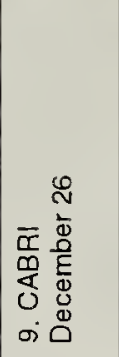 & 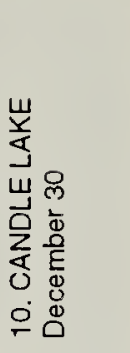 \\
\hline & & & & & & & & & \\
\hline & & + & & & & & & & \\
\hline & & & & & & & & & \\
\hline & & & & & & & & & \\
\hline . & & $T(2)$ & 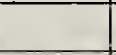 & $T(5)$ & $T$ & & & & $T(50+)$ \\
\hline & 9 & & 2 & $T(2)$ & $T(1)$ & & & & \\
\hline & & & & & & & & & \\
\hline & & & & & & & & & \\
\hline & & & & & & & & & \\
\hline & & & & & & & & & \\
\hline \multirow[t]{8}{*}{11} & & & & & $1+T(2)$ & & & & 10 \\
\hline & & & & & & & & & \\
\hline & & & & & & & & & \\
\hline & & + & & & & & & & \\
\hline & & & & & & & & & \\
\hline & & & & & & & & & \\
\hline & & & & & & & & & \\
\hline & & & & $T(14)$ & $T(4)$ & & & & $T(2)$ \\
\hline \multirow[t]{13}{*}{$\mathrm{T}$} & & + & & & $T(6)$ & & & & \\
\hline & & & & & & & & & \\
\hline & & & & & & & & & \\
\hline & & 1 & & & $T(4)$ & 1 & & & \\
\hline & 2 & 2 & 1 & 5 & $T(7)$ & & & 1 & \\
\hline & & & & & & & & & $T(1)$ \\
\hline & 10 & & & 2 & $T(5)$ & & & 2 & \\
\hline & & & & & & & & & \\
\hline & 2 & & & & & & & & \\
\hline & & & & & $T(2)$ & & & & \\
\hline & & & & & $T(2)$ & & & $\therefore$ & \\
\hline & & $T(1)$ & & 1 & $T(2)$ & & & & \\
\hline & & & & & $T(1)$ & & & & \\
\hline \multirow[t]{7}{*}{$T(1)$} & & & & & & & & & \\
\hline & & & & & $T(1)$ & & & & \\
\hline & & & & & & & & & \\
\hline & & & & & & & & & \\
\hline & & & & & & & & & $T(1)$ \\
\hline & & & & & & & & & \\
\hline & 6 & & & 2 & & & & $T$ & \\
\hline$T(10)$ & 68 & 8 & 18 & 6 & $T(4)$ & 4 & & & $1+T(100+)$ \\
\hline & & & & & & & & & \\
\hline$T(2)$ & & & & & & & & & $T(2)$ \\
\hline \multirow[t]{2}{*}{$T(4)$} & & & & & & & & & \\
\hline & 20 & & & & & & & & \\
\hline 6 & 7 & 8 & 3 & 8 & 13 & 2 & & 3 & 7 \\
\hline
\end{tabular}


Table 1-2: SASKATCHEWAN CHRISTMAS MAMMAL COUNTS

\section{SPECIES}

MASKED SHREW

SHREW species

EASTERN COTTONTAIL

NUTTALL'S COTTONTAIL

SNOWSHOE HARE

WHITE-TAILED JACK RABBIT

RICHARDSON'S GROUND SQUIRREL

BLACK-TAILED PRAIRIE DOG

GREY SQUIRREL

FOX SQUIRREL

AMERICAN RED SQUIRREL

NORTHERN FLYING SQUIRREL

AMERICAN BEAVER

DEER MOUSE

GAPPER'S RED-BACKED VOLE

\section{MUSKRAT}

MEADOW VOLE

VOLE species

MOUSE species

NORWAY RAT

HOUSE MOUSE

AMERICAN PORCUPINE

COYOTE

WOLF

RED FOX

SWIFT FOX

RACCOON

FISHER

ERMINE

LONG-TAILED WEASEL

LEAST WEASEL

WEASEL species

AMERICAN MINK

AMERICAN BADGER

STRIPED SKUNK

RIVER OTTER

BOBCAT

MULE DEER

WHITE-TAILED DEER

DEER species

MOOSE

AMERICAN ELK

PRONGHORN

TOTAL SPECIES

\begin{tabular}{|c|c|c|c|c|c|c|c|c|c|}
\hline \multicolumn{10}{|c|}{ LOCATION AND DATE } \\
\hline \multirow[t]{2}{*}{ 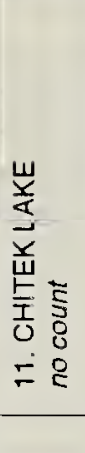 } & 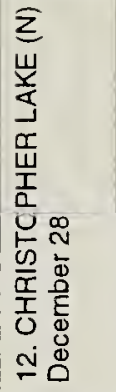 & 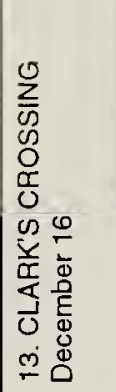 & 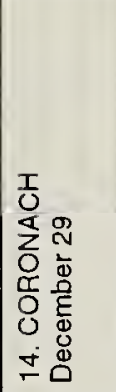 & 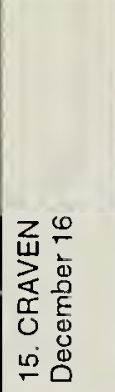 & 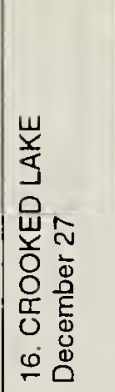 & 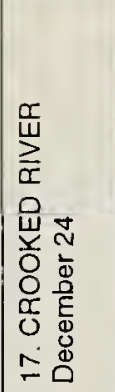 & 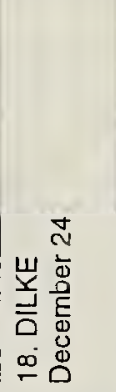 & 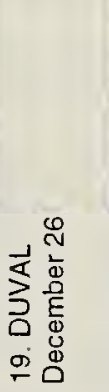 & 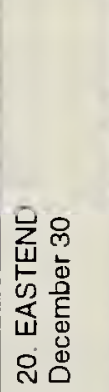 \\
\hline & & & $T(1)$ & & & & & & \\
\hline & & & & & & & & & \\
\hline & & & & & & & & & \\
\hline & & & $T(1)$ & & & & & & \\
\hline & $T(1)$ & $3+T(17)$ & & & $\mathrm{T}$ & $T(1)$ & & & \\
\hline & & 1 & $T(7)$ & & $\mathrm{T}$ & $T(1)$ & & $T(2)$ & \\
\hline & & & & & & & & & \\
\hline & & & & & & & & & \\
\hline & & & & 3 & & & & & \\
\hline & & & & & & & & & \\
\hline & 8 & & & 3 & 2 & 3 & & & \\
\hline & & & & & & & & & \\
\hline & $L(1)$ & & & & 3 & & & & \\
\hline & & & $T(2)$ & & & & & & \\
\hline & & & & & & & & & \\
\hline & $D(1)$ & & & & 3 & & & & \\
\hline & & & & & $\mathrm{T}$ & & & & \\
\hline & & 3 & $T(2)$ & & & & & & \\
\hline & $T(4)$ & $T(8)$ & & & $T$ & & & $T(4)$ & \\
\hline & & & & & & & & $T(1)$ & \\
\hline & & & & & & & & & \\
\hline & & $T(1)$ & & & & & & $T(1)$ & 2 \\
\hline & & $3+T(6)$ & 3 & 3 & 1 & $T(1)$ & & $H(2)$ & \\
\hline & + & & & & & & & & \\
\hline & $T(1)$ & $5+T(1)$ & $T(2)$ & 1 & $T$ & & & 1 & \\
\hline & & & & & & & & $T(1)$ & \\
\hline & & & & & & & & & \\
\hline & & & & & & & & & \\
\hline & & & & & & & & & \\
\hline & & & & & & & & $T(2)$ & \\
\hline & 1 & & & & & & & & \\
\hline & & $1+T(1)$ & & & & & & & \\
\hline & & & & & & & & & \\
\hline & & & & & & & & & \\
\hline & & & & & & & & $T(3)$ & \\
\hline & & & & & & & & & \\
\hline & & & & & & & & & \\
\hline & & 10 & 9 & 9 & & & & & 8 \\
\hline & $T(2)$ & 14 & 2 & 32 & 5 & 6 & 10 & 13 & 22 \\
\hline & & $T(10)$ & & & 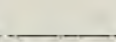 & & & $T(14)$ & \\
\hline & & & & & & & & & \\
\hline & & & & & & & & & \\
\hline & & & & & & & & & \\
\hline & 9 & 11 & 9 & 6 & 10 & 5 & 1 & 11 & 3 \\
\hline
\end{tabular}




\section{Table 1-3: SASKATCHEWAN CHRISTMAS MAMMAL COUNTS}

\section{SPECIES}

MASKED SHREW

SHREW species

EASTERN COTTONTAIL

NUTTALL'S COTTONTAIL

SNOWSHOE HARE

WHITE-TAILED JACK RABBIT

RICHARDSON'S GROUND SQUIRREL

BLACK-TAILED PRAIRIE DOG

GREY SQUIRREL

FOX SQUIRREL

AMERICAN RED SQUIRREL

NORTHERN FLYING SQUIRREL

AMERICAN BEAVER

DEER MOUSE

GAPPER'S RED-BACKED VOLE

MUSKRAT

MEADOW VOLE

VOLE species

MOUSE species

NORWAY RAT

HOUSE MOUSE

AMERICAN PORCUPINE

COYOTE

WOLF

RED FOX

SWIFT FOX

RACCOON

FISHER

ERMINE

LONG-TAILED WEASEL

LEAST WEASEL

WEASEL species

AMERICAN MINK

AMERICAN BADGER

STRIPED SKUNK

RIVER OTTER

BOBCAT

MULE DEER

WHITE-TAILED DEER

DEER species

MOOSE

AMERICAN ELK

PRONGHORN

TOTAL SPECIES

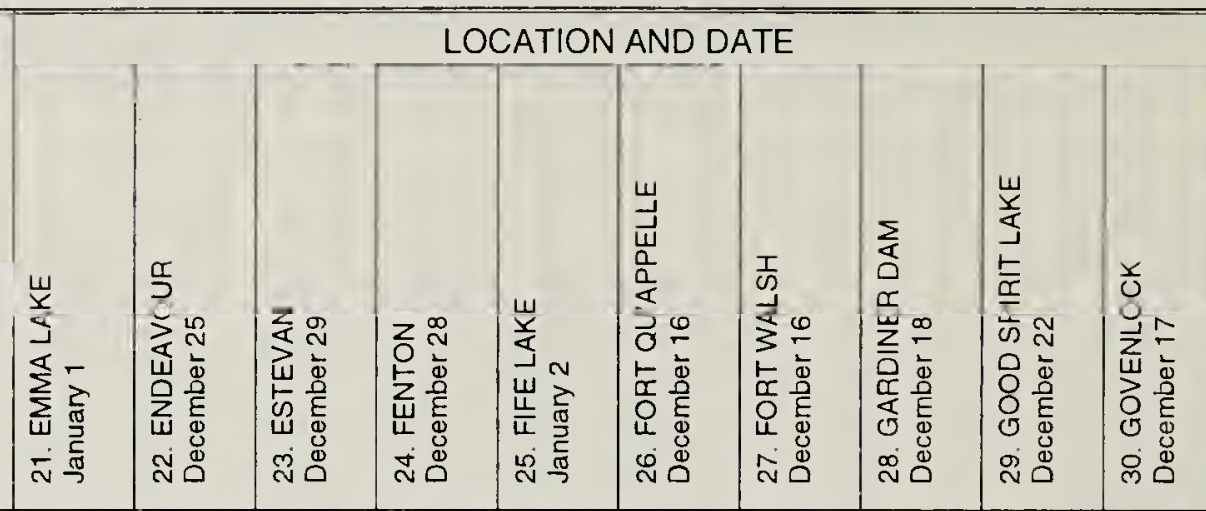

$$
\text { EL }
$$

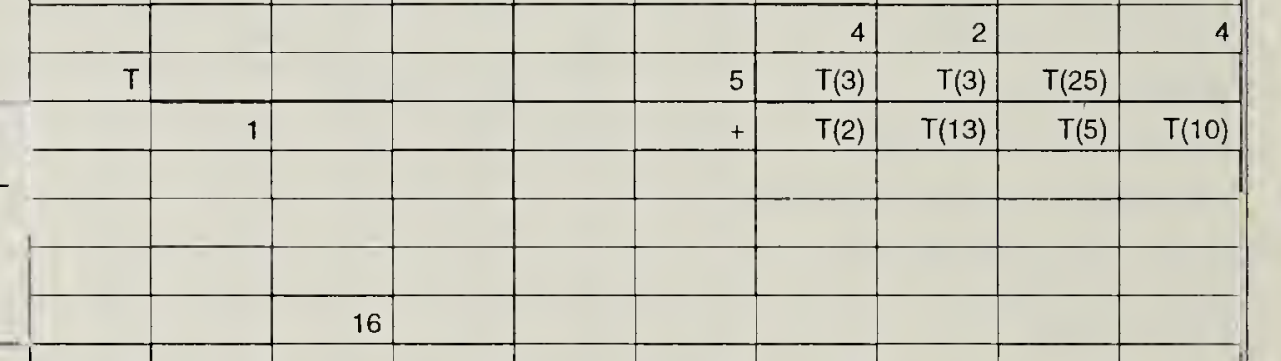

\begin{tabular}{|r|r|r|r|r|r|r|r|r|r|}
\hline 4 & 2 & & 3 & & 21 & 38 & & 5 & \\
\hline & & & & & & & & 2 & \\
\hline & & & & & & & & $\mathrm{L}(1)$ & \\
\hline & & & & & 1 & & & $\mathrm{~T}(2)$ & \\
\hline & & & & & & & & 2 \\
\hline
\end{tabular}


Table 1-4: SASKATCHEWAN CHRISTMAS MAMMAL COUNTS

SPECIES

MASKED SHREW

SHREW species

EASTERN COTTONTAIL

NUTTALL'S COTTONTAIL

SNOWSHOE HARE

WHITE-TAILED JACK RABBIT

RICHARDSON'S GROUND SQUIRREL

BLACK-TAILED PRAIRIE DOG

GREY SQUIRREL

FOX SQUIRREL

AMERICAN RED SQUIRREL

NORTHERN FLYING SQUIRREL

AMERICAN BEAVER

DEER MOUSE

GAPPER'S RED-BACKED VOLE

MUSKRAT

MEADOW VOLE

VOLE species

MOUSE species

NORWAY RAT

HOUSE MOUSE

AMERICAN PORCUPINE

COYOTE

WOLF

RED FOX

SWIFT FOX

RACCOON

FISHER

ERMINE

LONG-TAILED WEASEL

LEAST WEASEL

WEASEL species

AMERICAN MINK

AMERICAN BADGER

STRIPED SKUNK

RIVER OTTER

BOBCAT

MULE DEER

WHITE-TAILED DEER

DEER species

MOOSE

AMERICAN ELK

PRONGHORN

TOTAL SPECIES

\begin{tabular}{|c|c|c|c|c|c|c|c|c|c|}
\hline \multicolumn{10}{|c|}{ LOCATION AND DATE } \\
\hline 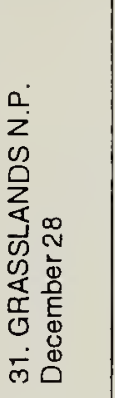 & 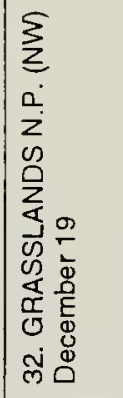 & 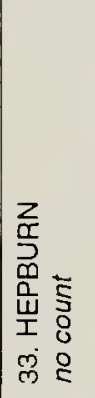 & 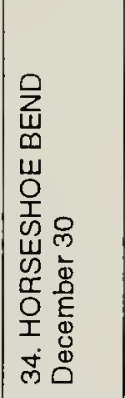 & 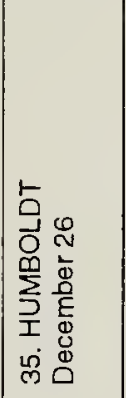 & 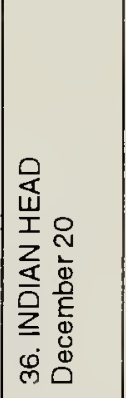 & 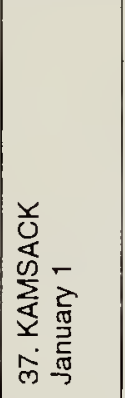 & 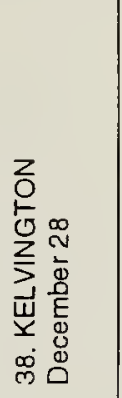 & 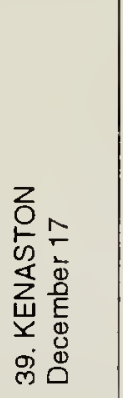 & 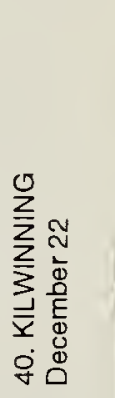 \\
\hline & & & & & & & & & \\
\hline & & & & + & & & $T(1)$ & & \\
\hline & & & & & & & & & \\
\hline \multirow[t]{2}{*}{1} & 2 & & & & & & & & \\
\hline & & & & $T(10)$ & 1 & 22 & $T(24)$ & $\mathrm{T}(25)$ & \\
\hline \multirow[t]{2}{*}{$T(2)$} & & & 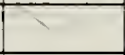 & $T(3)$ & & & $\mathrm{T}(6)$ & $T(8)$ & \\
\hline & & & & & & & & & \\
\hline \multicolumn{10}{|l|}{1} \\
\hline & & & & & & & & & \\
\hline & & & & & & & & & \\
\hline & & & & + & 16 & 8 & & & 3 \\
\hline & & & & & & & & & \\
\hline & & & & & & & & & \\
\hline & & & & & & & & & \\
\hline & & & & & & & & & \\
\hline & & & & $\mathrm{D}(1)$ & & & & & \\
\hline & & & & & & & & & \\
\hline & & & & & & & & & \\
\hline$T(20)$ & & & $T(5)$ & & & & $T(21)$ & $\mathrm{T}$ & \\
\hline & & & & & & & & & \\
\hline \multicolumn{10}{|l|}{14} \\
\hline \multirow[t]{4}{*}{1} & & & & $T(2)$ & & 1 & $D(1)+T(6)$ & & \\
\hline & 4 & & & $T(6)$ & 1 & $H(1)$ & $T(4)$ & $T(2)$ & 1 \\
\hline & & & & & & & & & \\
\hline & & & 1 & $T(3)$ & & 3! & $T(2)$ & 1 & $T$ \\
\hline \multicolumn{10}{|c|}{11} \\
\hline & & & & & & & + & & \\
\hline & & & & & & & & & \\
\hline & & & & & & & & & \\
\hline & & & & + & & 1 & & & \\
\hline & & & $T(1)$ & & & & & $T(3)$ & \\
\hline \multirow[t]{2}{*}{$T(1)$} & & & & & & & $T(6)$ & & \\
\hline & & & & + & & & & & \\
\hline \multicolumn{10}{|l|}{$d(1)$} \\
\hline & & & & & 1 & & & & \\
\hline & & & & & 1 & & & & \\
\hline & & & & & & & & & \\
\hline 28 & & & & & & & & 2 & \\
\hline \multirow[t]{4}{*}{7} & 6 & & 5 & $T(20)$ & 8 & 133 & $T(40)$ & 21 & $T(100+)$ \\
\hline & & & & & & & & & \\
\hline & & & & & & 7 & & & \\
\hline & & & & & & 25 & & & \\
\hline \multicolumn{10}{|c|}{15} \\
\hline 12 & 3 & & 4 & 11 & 6 & 9 & 10 & 8 & 4 \\
\hline
\end{tabular}




\section{Table 1-5: SASKATCHEWAN CHRISTMAS MAMMAL COUNTS}

\section{SPECIES}

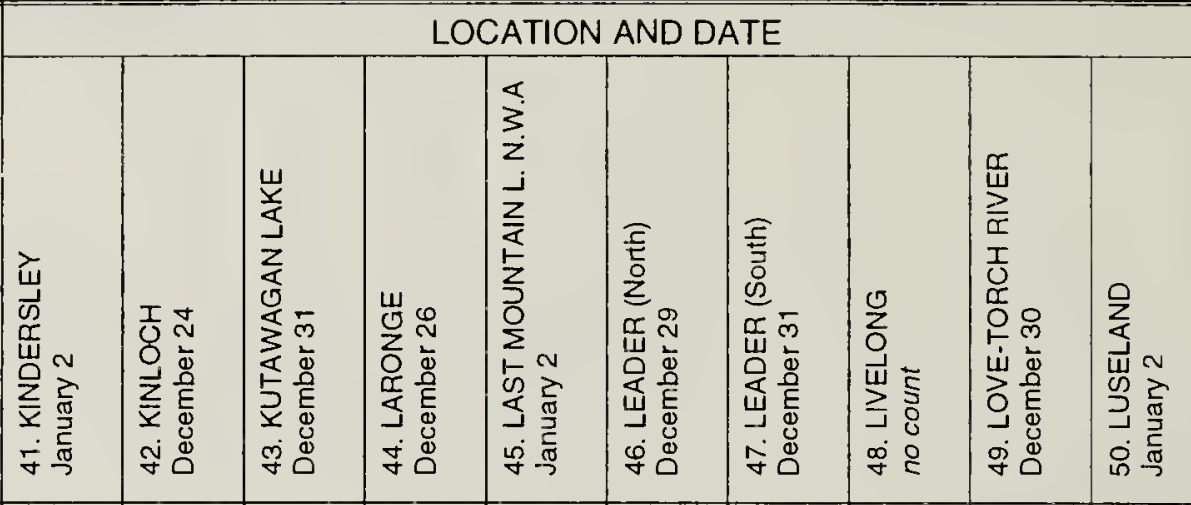

MASKED SHREW

SHREW species

EASTERN COTTONTAIL

NUTTALL'S COTTONTAIL

SNOWSHOE HARE.

WHITE-TAILED JACK RABBIT

RICHARDSON'S GROUND SQUIRREL

BLACK-TAILED PRAIRIE DOG

GREY SQUIRREL

FOX SQUIRREL

AMERICAN RED SQUIRREL

NORTHERN FLYING SQUIRREL

AMERICAN BEAVER

DEER MOUSE

GAPPER'S RED-BACKED VOLE

MUSKRAT

MEADOW VOLE

VOLE species

MOUSE species

NORWAY RAT

HOUSE MOUSE

AMERICAN PORCUPINE

COYOTE

WOLF

RED FOX

SWIFT FOX

RACCOON

FISHER

ERMINE

LONG-TAILED WEASEL

LEAST WEASEL

WEASEL species

AMERICAN MINK

AMERICAN BADGER

STRIPED SKUNK

RIVER OTTER

BOBCAT

MULE DEER

WHITE-TAILED DEER

DEER species

MOOSE

AMERICAN ELK

PRONGHORN

TOTAL SPECIES

\begin{tabular}{|r|r|r|r|r|r|r|r|r|r||}
\hline & & & & & & & & & \\
\hline & & & & & & & & & \\
\hline & & & & & & & & & \\
\hline & & & & & 2 & & & & \\
\hline & $\mathrm{T}(20)$ & $\mathrm{T}(1)$ & $\mathrm{T}(7)$ & & & & & & \\
\hline & & $\mathrm{T}(2)$ & & $\mathrm{T}$ & 2 & & & & 2 \\
\hline & & & & & & & & & \\
\hline
\end{tabular}

\begin{tabular}{|r|r|r|r|r|r|r|r|r|r||}
\hline & & & & & & & & & \\
\hline & & & & & & & & & \\
\hline & & & & & & & & & \\
\hline & & & 3 & & & & & 4 & \\
\hline & $\mathrm{L}(3)$ & & & $\mathrm{L}(1)$ & & & & & \\
\hline & & & & & & & & & 2 \\
\hline
\end{tabular}


Table 1-6: SASKATCHEWAN CHRISTMAS MAMMAL COUNTS

\section{SPECIES}

MASKED SHREW

SHREW species

EASTERN COTTONTAIL

NUTTALL'S COTTONTAIL

SNOWSHOE HARE

WHITE-TAILED JACK RABBIT

RICHARDSON'S GROUND SQUIRREL

BLACK-TAILED PRAIRIE DOG

GREY SQUIRAEL

FOX SQUIRREL

AMERICAN RED SQUIRREL

NORTHERN FLYING SQUIRREL

AMERICAN BEAVER

DEER MOUSE

GAPPER'S RED-BACKED VOLE

MUSKRAT

MEADOW VOLE

VOLE species

MOUSE species

NORWAY RAT

HOUSE MOUSE

AMERICAN PORCUPINE

COYOTE

WOLF

RED FOX

SWIFT FOX

RACCOON

FISHER

ERMINE

LONG-TAII_ED WEASEL

LEAST WEASEL

WEASEL species

AMERICAN MINK

AMERICAN BADGER

STRIPED SKUNK

RIVER OTTER

BOBCAT

MULE DEER

WHITE-TAILED DEER

DEER species

MOOSE

AMERICAN ELK

PRONGHORN

TOTAL SPECIES

\begin{tabular}{|c|c|c|c|c|c|c|c|c|c|}
\hline \multicolumn{10}{|c|}{ LOCATION AND DATE } \\
\hline 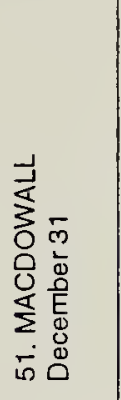 & 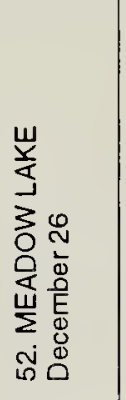 & 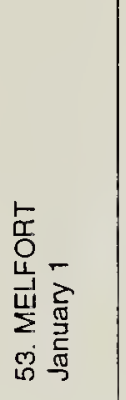 & 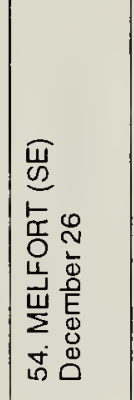 & 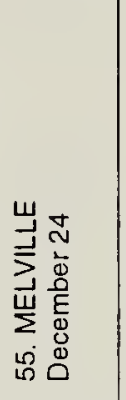 & 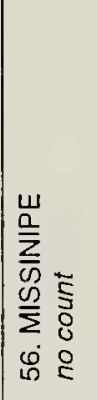 & 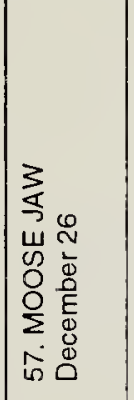 & 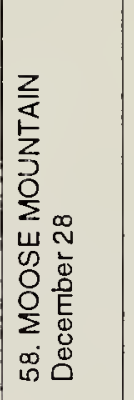 & 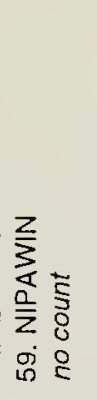 & 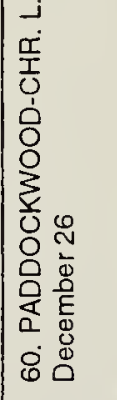 \\
\hline & & & & & & & & & \\
\hline & & & & & & & & & \\
\hline & & & & & & & & & \\
\hline & & & & & & 2 & & & \\
\hline & 1 & 12 & & & & & & & \\
\hline+ & & 2 & & & & 5 & & & \\
\hline & & & & & & & & & \\
\hline & & & & & & 4 & & & \\
\hline & & & & & & & & & \\
\hline & & & & & & 9 & & & \\
\hline 2 & 5 & & & & & & 19 & & 2 \\
\hline & & & & & & & & & \\
\hline & & & & & & 1 & & & \\
\hline & & & & & & & & & \\
\hline & & & & & & & & & \\
\hline & & & & $L(3)$ & & & & & \\
\hline & & & & & & & & & \\
\hline & & & & & & & & & \\
\hline $\mathrm{T}$ & & & & $T(2)$ & & & & & \\
\hline & & & & & & & & & \\
\hline & & & & & & & & & \\
\hline & 3 & & 1 & 1 & & & 3. & & \\
\hline$T(2)$ & 1 & 2 & 12 & 1 & & & $\rightarrow$ & & 1 \\
\hline & & & & & & & 1 & & \\
\hline$T(7)$ & & 2 & 3. & 1 & & 2 & 2. & & \\
\hline & & & & & & & & & \\
\hline$T(1)$ & & & & 1 & & & & & \\
\hline & & & & & & & & & \\
\hline & & & & & & & & & \\
\hline & & & & & & & & & \\
\hline$T(12)$ & & & & & & & & & \\
\hline & & $T(1)$ & & & & $T(1)$ & & & \\
\hline$T(2)$ & & & & & & $T(1)$ & & & \\
\hline & & & & & & $d(3)$ & & & \\
\hline & & & & & & & & & \\
\hline & & & & & & & & & \\
\hline & & & & & & & & & \\
\hline$T(4)$ & & 4 & & $1+T(10)$ & & 35 & 24 & & \\
\hline & & & & & & & & & \\
\hline & & & & & & & 1. & & \\
\hline & & & & & & & & & \\
\hline & & & & & & & & & \\
\hline 9 & 4 & 6 & 3 & 7 & & 9 & 5 & & 2 \\
\hline
\end{tabular}




\section{Table 1-7: SASKATCHEWAN CHRISTMAS MAMMAL COUNTS}

\section{SPECIES}

MASKED SHREW

SHREW species

EASTERN COTTONTAIL

NUTTALL'S COTTONTAIL

SNOWSHOE HARE

WHITE-TAILED JACK RABBIT

RICHARDSON'S GROUND SQUIRREL

BLACK-TAILED PRAIRIE DOG

GREY SOUIRREL

FOX SQUIRREL

AMERICAN RED SQUIRREL

NORTHERN FLYING SOUIRREL

AMERICAN BEAVER

DEER MOUSE

GAPPER'S RED-BACKED VOLE

MUSKRAT

MEADOW VOLE

VOLE species

MOUSE species

NORWAY RAT

HOUSE MOUSE

AMERICAN PORCUPINE

COYOTE

WOLF

RED FOX

SWIFT FOX

RACCOON

FISHER

ERMINE

LONG-TAILED WEASEL

LEAST WEASEL

WEASEL species

AMERICAN MINK

AMERICAN BADGER

STRIPED SKUNK

RIVER OTTER

BOBCAT

MULE DEER

WHITE-TAILED DEER

DEER species

MOOSE

AMERICAN ELK

PRONGHORN

TOTAL SPECIES

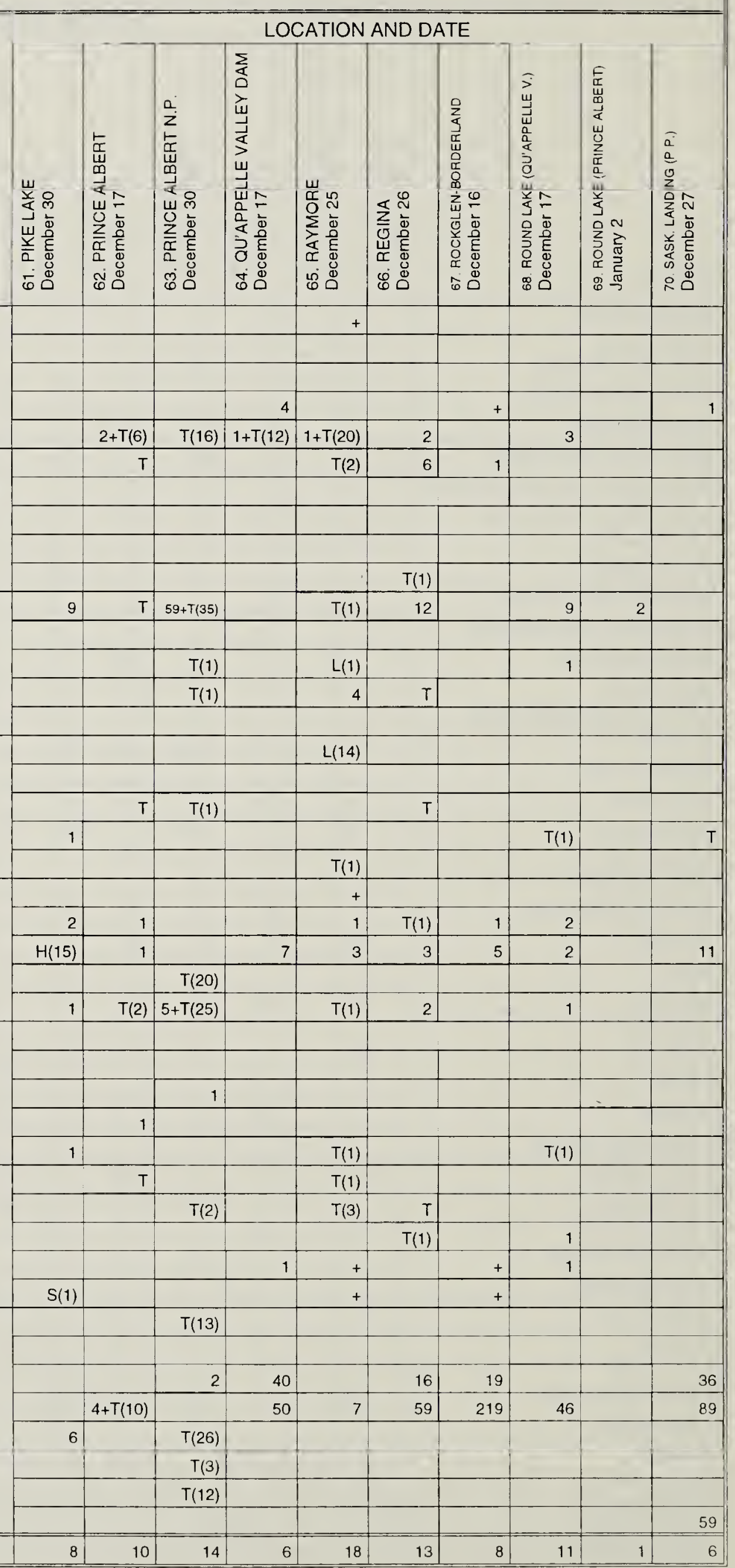


Table 1-8: SASKATCHEWAN CHRISTMAS MAMMAL COUNTS

\section{SPECIES}

MASKED SHREW

SHREW species

EASTERN COTTONTAIL

NUTTALL'S COTTONTAIL

SNOWSHOE HARE

WHITE-TAILED JACK RABBIT

RICHARDSON'S GROUND SQUIRREL

BLACK-TAILED PRAIRIE DOG

GREY SQUIRREL

FOX SQUIRREL

AMERICAN RED SQUIRREL

NORTHERN FLYING SQUIRREL

AMERICAN BEAVER

DEER MOUSE

GAPPER'S RED-BACKED VOLE

MUSKRAT

MEADOW VOLE

VOLE species

MOUSE species

NORWAY RAT

HOUSE MOUSE

AMERICAN PORCUPINE

COYOTE

WOLF

RED FOX

SWIFT FOX

RACCOON

FISHER

ERMINE

LONG-TAILED WEASEL

LEAST WEASEL

WEASEL species

AMERICAN MINK

AMERICAN BADGER

STRIPED SKUNK

RIVER OTTER

BOBCAT

MULE DEER

WHITE-TAILED DEER

DEER species

MOOSE

AMERICAN ELK

PRONGHORN

TOTAL SPECIES

\begin{tabular}{|c|c|c|c|c|c|c|c|c|c|}
\hline \multicolumn{10}{|c|}{ LOCATION AND DATE } \\
\hline \multirow[t]{2}{*}{ 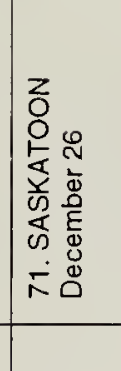 } & \multirow[t]{2}{*}{ 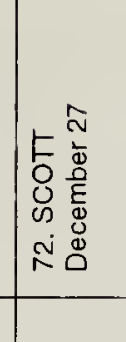 } & 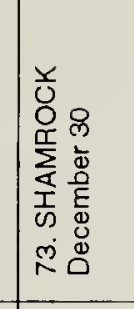 & 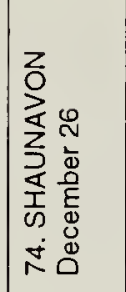 & 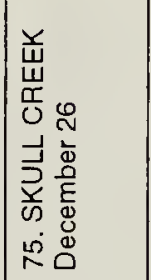 & 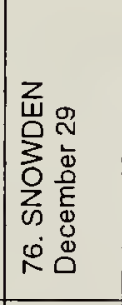 & 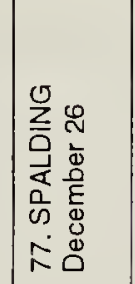 & 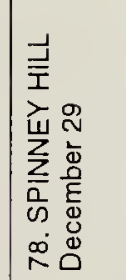 & 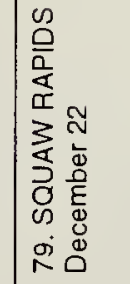 & 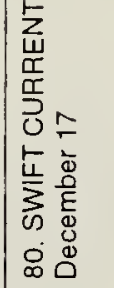 \\
\hline & & & & 1 & & & & & \\
\hline \multirow[t]{3}{*}{$T(1)$} & & & & & & T & & & ' \\
\hline & & & & & & & & & \\
\hline & & & & $27+T(390)$ & & & & & $5+T(3)$ \\
\hline $2+T(21)$ & $T(7)$ & & $\mathrm{x}$ & 10 & $T(15)$ & $\mathrm{T}$ & & $T(5)$ & $T(5)$ \\
\hline \multirow[t]{5}{*}{6} & $T(5)$ & 2 & 2 & $10+T(31)$ & + & & $T$ & & 1 \\
\hline & & & & 1 & & & & & \\
\hline & & & & & & & & & \\
\hline & & & & & & $\mathrm{T}$ & & & 5 \\
\hline & & & & & & & & & \\
\hline \multirow[t]{2}{*}{2} & & & & 4 & 42 & & 2 & 24 & \\
\hline & & & & & & & & & \\
\hline \multicolumn{10}{|l|}{$T(1)$} \\
\hline \multirow[t]{2}{*}{$T(11)$} & & & & $T(100+)$ & & & & & \\
\hline & & & & & & & & & \\
\hline \multirow[t]{2}{*}{1.} & & & & & & $L(9)$ & & & \\
\hline & & & & $T(100+)$ & & & $\mathrm{T}$ & & \\
\hline \multirow[t]{4}{*}{$T(2)$} & $T(2)$ & & & & & T & & $T(10)$ & \\
\hline & & & & & $T(7)$ & & & & \\
\hline & & & & & $T(1)$ & & & & \\
\hline & & & & & & & & & \\
\hline 1 & $T(1)$ & & & 2 & + & & 2 & & \\
\hline \multirow[t]{2}{*}{$8+T(3)$} & 1 & & 1 & $19+T(65)$ & 2 & $\mathrm{~T}$ & 2 & 1 & 4 \\
\hline & & & & & & & & $T(3)$ & \\
\hline \multirow[t]{6}{*}{$1+T(3)$} & $T(1)$ & $\mathrm{T}$ & 1 & $T(1)$ & $T(3)$ & $\mathrm{T}$ & & 1 & 1 \\
\hline & & & & & & & & & \\
\hline & & & & $T(2)$ & & & & & \\
\hline & & & & & & & & & \\
\hline & & & & & & & & & \\
\hline & & & & $4+T(6)$ & + & & & & \\
\hline$T(1)$ & & & & $T(2)$ & 1 & & & & \\
\hline \multirow[t]{4}{*}{$T(1)$} & $T(2)$ & & & & & $\mathrm{T}$ & & $T(2)$ & \\
\hline & & & & $T(3)$ & & & & & \\
\hline & & & & & & & & & \\
\hline & & & & 4 & & 1 & & & \\
\hline & & & & & & & & & \\
\hline & & & & & & & & & \\
\hline $15+T(1)$ & & & & 16 & & & & & 52 \\
\hline $29+T(11)$ & $T(5)$ & & & 177 & 6 & $\mathrm{~T}$ & 6 & $T(3)$ & 36 \\
\hline$T(13)$ & & $\mathrm{T}$ & & & & & & & \\
\hline & & & & & + & & & & \\
\hline & & & & & + & & & $T(10)$ & \\
\hline & & & & 40 & & & & & \\
\hline 14 & 8 & 3 & 3 & 19 & 13 & 10 & 6 & 9 & 8 \\
\hline
\end{tabular}


Table 1-9: SASKATCHEWAN CHRISTMAS MAMMAL COUNTS

\section{SPECIES}

MASKED SHREW

SHREW species

EASTERN COTTONTAIL

NUTTALL'S COTTONTAIL

SNOWSHOE HARE

WHITE-TAILED JACK RABBIT

RICHARDSON'S GROUND SQUIRREL BLACK-TAILED PRAIRIE DOG GREY SQUIRREL

FOX SQUIRREL

AMERICAN RED SQUIRREL

NORTHERN FLYING SQUIRREL.

AMERICAN BEAVER

DEER MOUSE

GAPPER'S RED-BACKED VOLE

MUSKRAT

MEADOW VOLE

VOLE species

MOUSE species

NORWAY RAT

HOUSE MOUSE

AMERICAN PORCUPINE

COYOTE

WOLF

RED FOX

SWIFT FOX

RACCOON

FISHER

ERMINE

LONG-TAILED WEASEL

LEAST WEASEL

WEASEL species

AMERICAN MINK

AMERICAN BADGER

STRIPED SKUNK

RIVER OTTER

BOBCAT

MULE DEER

WHITE-TAILED DEER

DEER species

MOOSE

AMERICAN ELK

PRONGHORN

TOTAL SPECIES

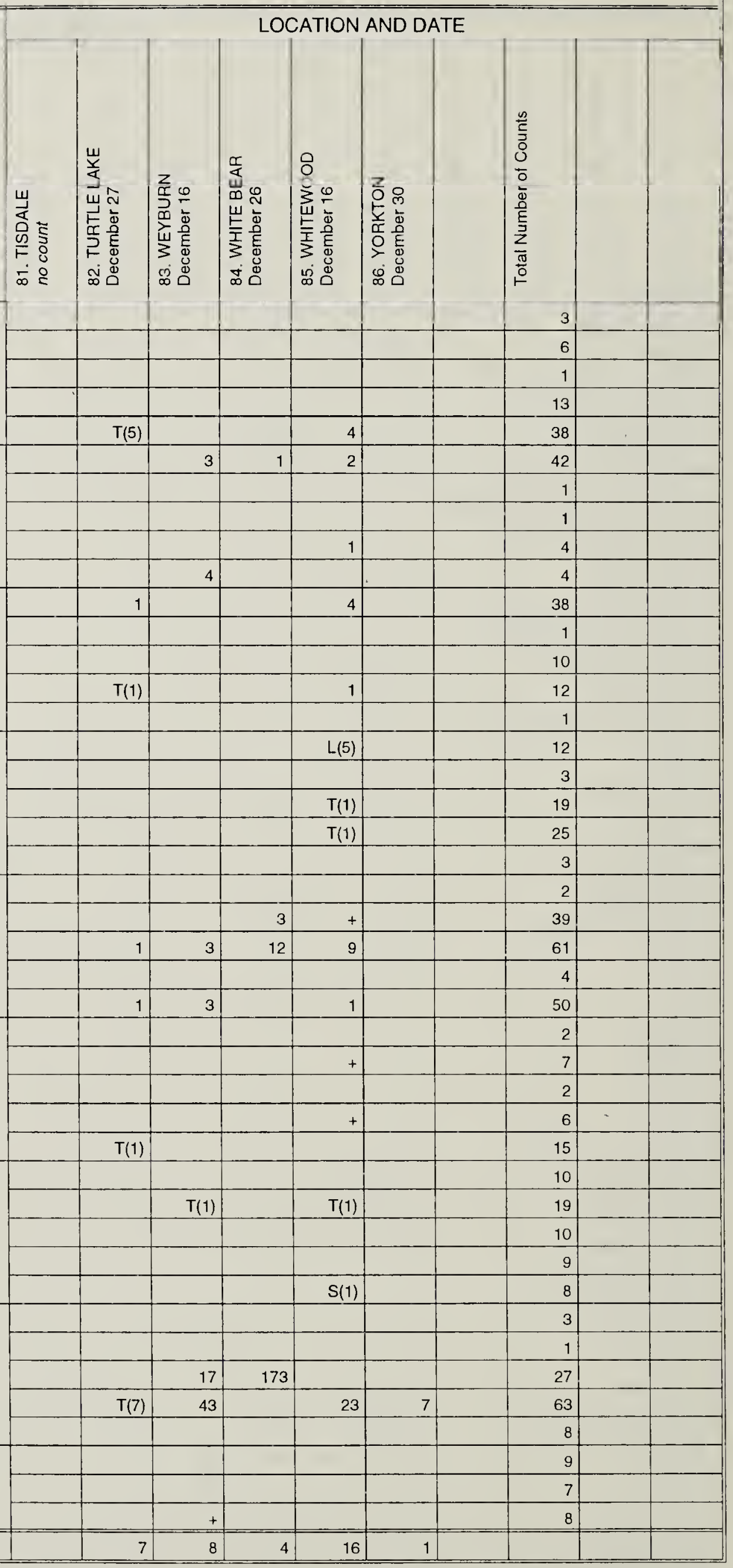

